Check for updates

Cite this: RSC Adv., 2017, 7, 56137

\section{Unravelling vaginal microbial genetic diversity and abundance between Holstein and Fleckvieh cattle}

\begin{abstract}
Lucky T. Nesengani, $\mathbb{D} \dagger$ Jun Wang, $\uparrow$ Yujiang Yang, Lianyu Yang and Wenfa Lu*
Characterization of bovine vaginal microbial genetic diversity can lead to better understanding of cattle physiology, which is of economic interest in improving cattle reproduction health. However, there is limited knowledge on the factors affecting the microbial community harbored in the vaginal tract. The aim of the current study is to evaluate and compare the microbial genetic diversity and abundance between the Holstein and Fleckvieh cattle using metagenomic approach. The main bacterial phyla found were firmicutes (51.70-58.80\%), proteobacteria (16.70-11.70\%) and bacteroidetes $(13.20-19.50 \%)$ for Holstein and Fleckvieh. Notable significant difference $(p<0.05)$ was observed for Fusobacteria, which was more abundant in Holstein (9.73-0.81\%). At the achaea phyla, Euryarchaeota was significantly different $(p<0.05)$ and was more abundant in Fleckvieh than in Holstein cattle $(0.86-0.22 \%)$. At the genus level, Turicibacter, Ruminococcaceae_UCG-010, Clostridium_sensu_stricto_1, Dorea, Methanobrevibacter, Acetitomaculum, Ruminococcaceae_NK4A214_group and Ruminiclostridium_5 were found to be significantly $(p<0.05)$ different between the two cattle breeds and all of them were more abundant in Fleckvieh than in Holstein cattle. The current results indicate that the breed can influence bovine vaginal microbial abundance and diversity. Our study characterized and provided pivotal scientific knowledge to understand the microbial community that harbors the bovine virginal flora.
\end{abstract}

Received 23rd September 2017 Accepted 29th November 2017 DOI: 10.1039/c7ra10553c rsc.li/rsc-advances metagenomics approach. ${ }^{17,18}$ Previous studies have shown that vaginal bacterial abundance and diversity in humans is shaped by different factors, such as pregnancy. ${ }^{6}$ Moreover, factors such as biogeographical and ethnic differences have been observed to influence vaginal bacterial abundance during pregnancy and postpartum in humans. ${ }^{19}$ However, knowledge of factors that influence vaginal bacterial genetic diversity and abundance in cattle is limited, particularly on a breed effect level.

Few studies have evaluated the cow vaginal microbial community. Till date, a study has evaluated the composition of vaginal microbial genetic diversity in the Nellore cattle using the metagenomic approach without taking into account the breed effect. ${ }^{20}$ Hence, the current study aims at evaluating the breed effect on the vaginal microbial diversity and abundance by describing and comparing the vaginal microbiomes structure of the Holstein and Fleckvieh cattle.

\section{Materials and methods}

\section{Animals and sample collections}

An elite herd (Guangyuan farm, Jilin Province, People's Republic of China) was selected, which is composed of Hereford and Fleckvieh cattle breed. Within the herd, a total of 12 cattle were randomly chosen from Holstein $(n=6)$ and Fleckvieh $(n=$ 6) cattle. The cattle considered in the current study were all reared under the same conditions. All animals were healthy and did not receive any antibiotic treatment in a month prior to 
sampling. Vaginal samples were collected from all 12 cows using swabs and stored at $-80{ }^{\circ} \mathrm{C}$ in a refrigerator until further use. This study was performed at the College of Animal Science and Technology, Jilin Agricultural University. All animal studies were conducted following the guidelines and regulations for the care and use of experimental animals established by the Ministry of Agriculture, China. Ethical approval for the present study was obtained from the Ethical Committee of Jilin Agricultural University, China.

\section{Total genomic DNA extraction and 16S rDNA gene amplification}

Total microbial genomic DNA was extracted from the swab of each sample using a QIAamp DNA mini kit (Qiagen, Valencia, CA, USA) following the manufacturer's recommendation. DNA concentration was evaluated by optimal density using NanoDrop 2000 (Thermo Electron Corporation, USA) at wavelengths of 260 and $280 \mathrm{~nm}$. The integrity of the DNA was assessed by electrophoresis on $1.0 \%$ agarose gels and visualized with E-Gel Imager (Tanon-2500, Shanghai, China). V4 hypervariable regions of $16 \mathrm{~S}$ rDNA were PCR amplified from microbial genomic DNA harvested from the vaginal samples. The barcoded fusion forward primer was $515 \mathrm{~F} 5^{\prime}$ GTGCCAGCMGCGGTAA-3' and the reverse primer was 806R 5'GGACTACHVGGGTATCTAAT- ${ }^{\prime}$. The PCR reactions were carried out in $30 \mu \mathrm{L}$ reactions with $15 \mu \mathrm{L}$ of Phusion ${ }^{\circledR}$ HighFidelity PCR MasterMix (New England Biolabs), $0.2 \mu \mathrm{M}$ of forward and reverse primers, and $10 \mathrm{ng}$ template DNA. The PCR conditions were as follows: initial denaturation at $98{ }^{\circ} \mathrm{C}$ for $1 \mathrm{~min}$, followed by 30 cycles of denaturation at $98{ }^{\circ} \mathrm{C}$ for $10 \mathrm{~s}$, annealing at $50{ }^{\circ} \mathrm{C}$ for $30 \mathrm{~s}$, and elongation at $72{ }^{\circ} \mathrm{C}$ for $30 \mathrm{~s}$ and post elongation at $72{ }^{\circ} \mathrm{C}$ for $5 \mathrm{~min}$. The PCR amplicon products were separated on $2 \%$ agarose gels and extracted from the gels. Samples with bright main strip between 200-450 bp were chosen for further experiment. PCR products were purified with GeneJET Gel Extraction Kit (Thermo Scientific, Waltham, USA).

\section{Library preparation and sequencing}

Sequencing libraries were generated using Illumina TruSeq DNA PCR-Free Library Preparation Kit (Illumina, USA) following manufacturer's recommendations and index codes were added. The library quality was assessed on the Qubit 2.0 Fluorometer (Thermo Scientific, Waltham, USA) and Agilent Bioanalyzer 2100 system. The library was sequenced on an Illumina HiSeq platform, and $250 \mathrm{bp}$ paired-end reads were generated.

\section{Data analysis}

The paired-end reads from the DNA fragments were merged using FLASH, ${ }^{21}$ which is an accurate analysis tool designed to merge paired-end reads when there are overlaps between the reads. Reads were first assigned to each sample according to the unique barcodes. Sequences were analyzed using QIIME ${ }^{22}$ software package (Quantitative Insights into Microbial Ecology). In-house perl scripts were used to analyze alpha-(within samples) and beta-(among samples), and reads were filtered by QIIME quality filters. Pick_de_novo_otus.py was used to pick operational taxonomic units (OTUs). Sequences with $\geq 97 \%$ similarity were assigned to the same OTUs. A representative of sequences for each OTU was classified using the RDP classifier ${ }^{23}$ to annotate taxonomic information for each representative sequence. All other statistical analyses were carried out using R, version 3.2.0. ${ }^{24}$ To compute alpha diversity, the OTU was rarified and metrics were calculated; chao1, Simpson index, ACE, goods coverage and Shannon's diversity index were generated based on these metrics. Both the weighted and unweighted unifrac that are phylogenetic measures of beta diversity were calculated. We used weighted unifrac for principal coordinate analysis (PCA) with arithmetic mean (UPGMA) clustering. The significant differences were tested at $p<0.05$ and $p<0.01$. The difference between the two groups was accessed by analysis of similarity (ANOSIM) in R Package "vegan". Heatmaps were generated using gplot in $\mathrm{R}$ with taxonomic data. A Venn diagram was generated to compare unique and common OTUs between the two cattle breeds using R package "VennDiagram".

\section{Results}

\section{Sequencing overview}

Twelve cattle ( $n=6$ for Holstein and $n=6$ for Fleckvieh) were sampled to evaluate and compare the vaginal microbial genetic diversity and abundance. Samples were used to generate V4 16S rDNA gene profiles. A total of 906914 reads were obtained in all samples with an average number of 75576 reads per sample.

\section{Significant difference between Holstein and Fleckvieh cattle}

Analysis of the phylogenetic composition of vaginal bacterial community between Holstein and Fleckvieh cattle was characterized by $16 \mathrm{~S}$ rDNA gene sequencing and the alpha- and betadiversity of microbiota across the two cattle breeds were compared. The data indicated a diverse structure, which is summarized in Table 1, indicating the diversity and abundance indexes (chao1, Shannon diversity index, and ACE and Simpson index).

The Fleckvieh cattle had a significantly higher Shannon index compared to the Holstein cattle $(p<0.05$, Fig. 1A). There was a clear variation in data distribution between the two groups. The significant difference between the two groups was observed using ANOSIM analysis ( $p<0.05$, Fig. 1B). The variation of phylogenetic diversity of the tested samples was evaluated. There was a slight mixture in clustering, in which sample F2 and F5 from the Fleckvieh were clustered into the samples from the Holstein group and sample H6 was clustered into the samples from Fleckvieh group in principal component analysis (PCA) (Fig. 1C). However, significant difference between the two clustered groups was observed ( $p<0.05$, Fig. 1C). Most of the samples indicated higher similarity in phylogenetic composition between the samples from the two groups when analysing the alpha-diversity. However, few of the samples indicated a slight distinct phylogenetic composition between the Holstein and Fleckvieh samples (Fig. 1D). Greater variation between the two breeds was observed while analysing the beta-diversity, the $t$-test bars for the significant difference between the two groups 
Table 1 Indicating the diversity and abundance indexes; chao1, Shannon diversity index, and ACE and Simpson index with goods coverage ${ }^{a}$

\begin{tabular}{|c|c|c|c|c|c|c|}
\hline Sample name & Observed species & Shannon & Simpson & Chao1 & $\mathrm{ACE}$ & Goods coverage \\
\hline $\mathrm{H} 1$ & 1573 & 7.924 & 0.983 & 1716.5 & 1738.151 & 0.996 \\
\hline $\mathrm{H} 2$ & 1402 & 6.319 & 0.931 & 1552.785 & 1560.483 & 0.996 \\
\hline H3 & 1473 & 8.126 & 0.987 & 1581.247 & 1592.57 & 0.997 \\
\hline $\mathrm{H} 4$ & 1338 & 7.482 & 0.979 & 1428.017 & 1438.394 & 0.997 \\
\hline H5 & 1610 & 7.97 & 0.982 & 1739.814 & 1754.923 & 0.996 \\
\hline H6 & 1373 & 6.963 & 0.907 & 1447.072 & 1443.439 & 0.998 \\
\hline F1 & 1553 & 8.415 & 0.992 & 1658.174 & 1663.541 & 0.997 \\
\hline $\mathrm{F} 2$ & 1602 & 8.547 & 0.992 & 1711.48 & 1710.816 & 0.997 \\
\hline F3 & 1342 & 8.62 & 0.994 & 1395.382 & 1404.364 & 0.998 \\
\hline F4 & 1481 & 8.573 & 0.993 & 1598.56 & 1580.378 & 0.997 \\
\hline F5 & 1462 & 6.595 & 0.877 & 1554.557 & 1561.573 & 0.997 \\
\hline F6 & 1785 & 8.26 & 0.981 & 2412.338 & 2122.975 & 0.993 \\
\hline
\end{tabular}

${ }^{a} \mathrm{H} 1$ to $\mathrm{H} 6$ refers to the samples per individual from the Holstein cattle and F1 to F6 refers to the samples from the Fleckvieh cattle group.
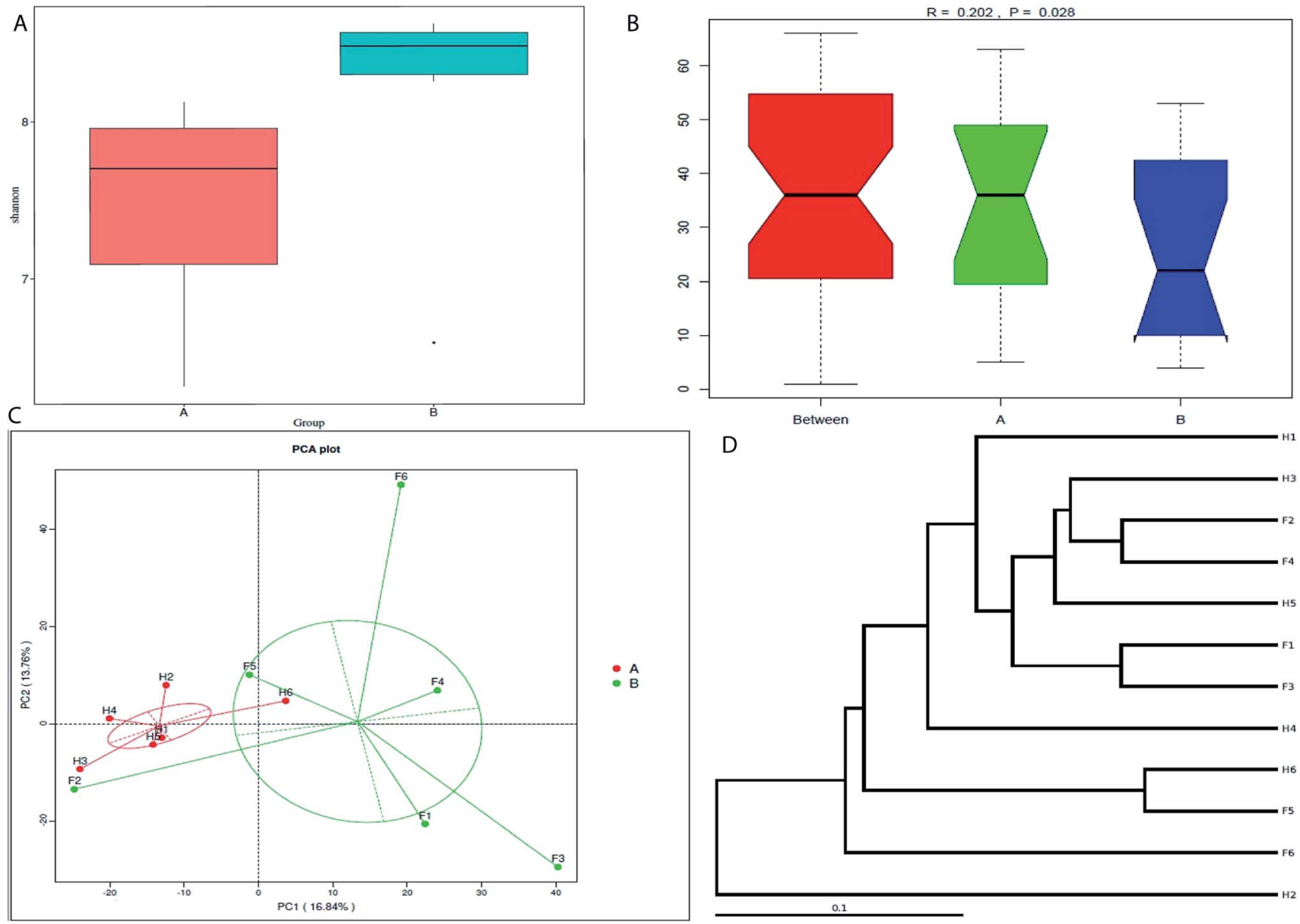

Fig. 1 Alpha- and beta-diversity comparison of the vaginal microbiomes of the Holstein and Fleckvieh cattle breeds. The analyses were performed on 16S rRNA V4 region data. The coding names are as follows: group A refers to the Holstein cattle group and group B refers to the Fleckvieh cattle group. $\mathrm{H} 1$ to $\mathrm{H} 6$ refers to the samples per individual from the Holstein cattle and $\mathrm{F} 1$ to F6 refers to the samples from the Fleckvieh cattle group. (A) Alpha-diversity comparison based on Shannon's diversity index, grouped by sampling cattle breed (mean \pm SEM). Compared to the Holstein, the Fleckvieh had a higher microbial richness. (B) Analysis of similarities between the two groups (Holstein and Fleckvieh). (C) Principal component analysis of vaginal bacterial microflora from two groups. (D) Weighted_unifrac cluster tree. Samples indicated a mixture of cluster with some from Holstein tending to cluster with Fleckvieh.

at the phylum level are reported in Fig. 2A. Fusobacteria and Euryarchaeota were the notable significant differences between the two groups at the phylum level. Fusobacteria was more abundant in the Holstein cattle, while the Euryarchaeota was more abundant in the Fleckvieh cattle. The significant difference at the genus level is reported in Fig. 3B; the 
A

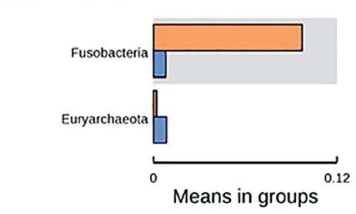

$B \square \mathrm{A} \square_{B}$

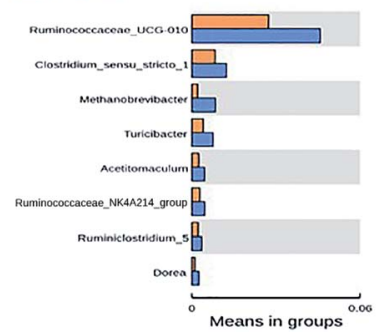

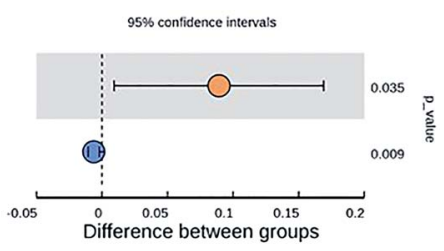

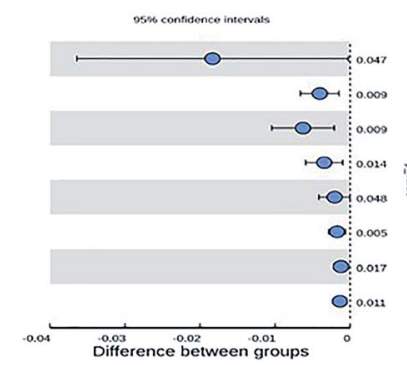

Fig. 2 Beta-diversity (among samples) comparison of the vaginal microbiomes of the Holstein and Fleckvieh cattle breeds. The t-test bars plot indicate significant difference between the two groups. (A) Beta-diversity comparison based on $t$-test significance results at the phylum level. (B) Beta-diversity comparison based on $t$-test significance results at the genus level.

Ruminococcaceae_UCG_010 amount showed significant difference between the two groups ( $p<0.05$, Fig. 2B). It was more abundant in the Fleckvieh than in the Holstein cattle. The most significant differences ( $p<0.01$, Fig. 2B) at the genus level were in Clostridium_sensu_stricto_1, Methanobrevibacter, Turicibacter, Dorea, Ruminiclostridium_5 and Ruminococcaceae_NK4A214_group. All the significant differences at the genus level were more abundant in the Fleckvieh cattle than in the Holstein cattle.

\section{Core bacterial abundance between the cattle breeds}

The taxonomic distribution of the numerically abundant bacteria derived from the 16S rRNA gene sequences was further evaluated. At the phylum level, firmicutes was the highest abundant phenotype in the Holstein and Fleckvieh group, which accounted for $51.70 \%$ and $58.80 \%$ of relative abundance respectively, followed by proteobacteria that accounted for $16.70 \%$ and $11.70 \%$ of relative abundance and bacteroidetes that accounted for $13.20 \%$ and $19.50 \%$ of relative abundance (Fig. 3A). Conversely, bacteria abundance was further evaluated using a heat map analysis for the top 26 bacteria at phylum level (Fig. 3B), which indicated similar results as previously explained with Fusobacteria indicating to be more abundant in the Holstein group and less abundant in the Fleckvieh group, while others indicated slight variation between the two groups at a similar magnitude. At the genus level, Actinobacillus had $4.70 \%$ abundance in the Holstein group, but only $0.06 \%$ abundance in the Fleckvieh group; however, there was no significant difference $(p>0.05)$. Moreover, it should be noted that the Facklamia and Histophilus accounted for $2.3 \%$ and $3.8 \%$ of abundance in the Holstein, while they accounted for $0.03 \%$ and $0.07 \%$ of abundance in the Fleckvieh group respectively ( $p>$ 0.05 , Fig. 3C).

\section{Common and unique operational taxonomic units (OTU)}

The common and unique operational taxonomic units (OTUs) between the two groups were evaluated. A total of 2574 OTUs were observed from all the samples. About 348 OTUs were identified to be unique in Fleckvieh cattle, while a total of 151 OTUs were identified to be unique in Holstein cattle; in addition, 2075 of OTUs exist in both groups and are defined as the core (Fig. 4A). Furthermore, the OTUs were evaluated based on each sample. Samples from the Fleckvieh cattle were found to have 22 OTUs and above, while the samples from Holstein were found to contain 18 OTUs and below with the only exception of H6 sample that contained 24 OTUs (Fig. 4B).

\section{Discussion}

Bovine microbial composition has been fairly studied primarily in the gut with few studies in the vaginal microbial composition. ${ }^{\mathbf{1 4 2 5 , 2 6}}$ To the best of our knowledge, there is no report based on bovine metagenomic studies that evaluated the vaginal microbial genetic diversity and abundance between cattle breeds. Cattle vaginal microbiota has been evaluated primarily based on the effects of diseases through culture-depended technique.,9,27 In this study, we compared vaginal microbial community structure between the Holstein and the Fleckvieh cattle breeds. The two breeds were chosen since Holstein is the most common and leading breed in dairy breeds across the world and in China, while the Fleckvieh is the common breed used for beef in Northern China.

Bacterial composition and diversity are indicated to be breed type-dependent between the two groups (Holstein and Fleckvieh cattle). The results of the bacterial composition are comparable to the study conducted by Laguardia-Nascimento et al. ${ }^{20}$ The group evaluated vaginal microbiota composition in Nellore cattle and found that firmicutes (40-50\%) was predominant in the vaginal microbiota, followed by bacteroidetes (15-25\%) and proteobacteria (5-25\%). Swarts et al. ${ }^{7}$ also reported that the vaginal microbiota of ewes and dairy cows is predominated by bacteroidetes, followed by Fusobacteria and proteobacteria. The studies conducted to evaluate bovine vaginal microbiota are in general agreement with the current study. Almost all the studies reported that the bovine vaginal microbiota is predominated by similar groups of bacteria (firmicutes, bacteroidetes and proteobacteria) at different magnitudes, which could be due to environmental factors and host genotype. However, it is also worthy to note that similar microbiota composition has also been observed in other sites, such as in the gut. Jami et al. ${ }^{28}$ found that bovine microbial composition in the gut is predominated by bacteroidetes, which is followed by firmicutes and proteobacteria at $51 \%, 41 \%$ and $5.46 \%$ respectively.

In the current study, it was found that different breeds dictate different bacterial genetic composition, in which Holstein cattle have less abundant and diverse bacteria than the Fleckvieh cattle. Greater bacterial genetic diversity has been associated with less risk of infections in dairy cattle. ${ }^{29}$ It was also noted that Fusobacteria was more abundant in the Holstein than 

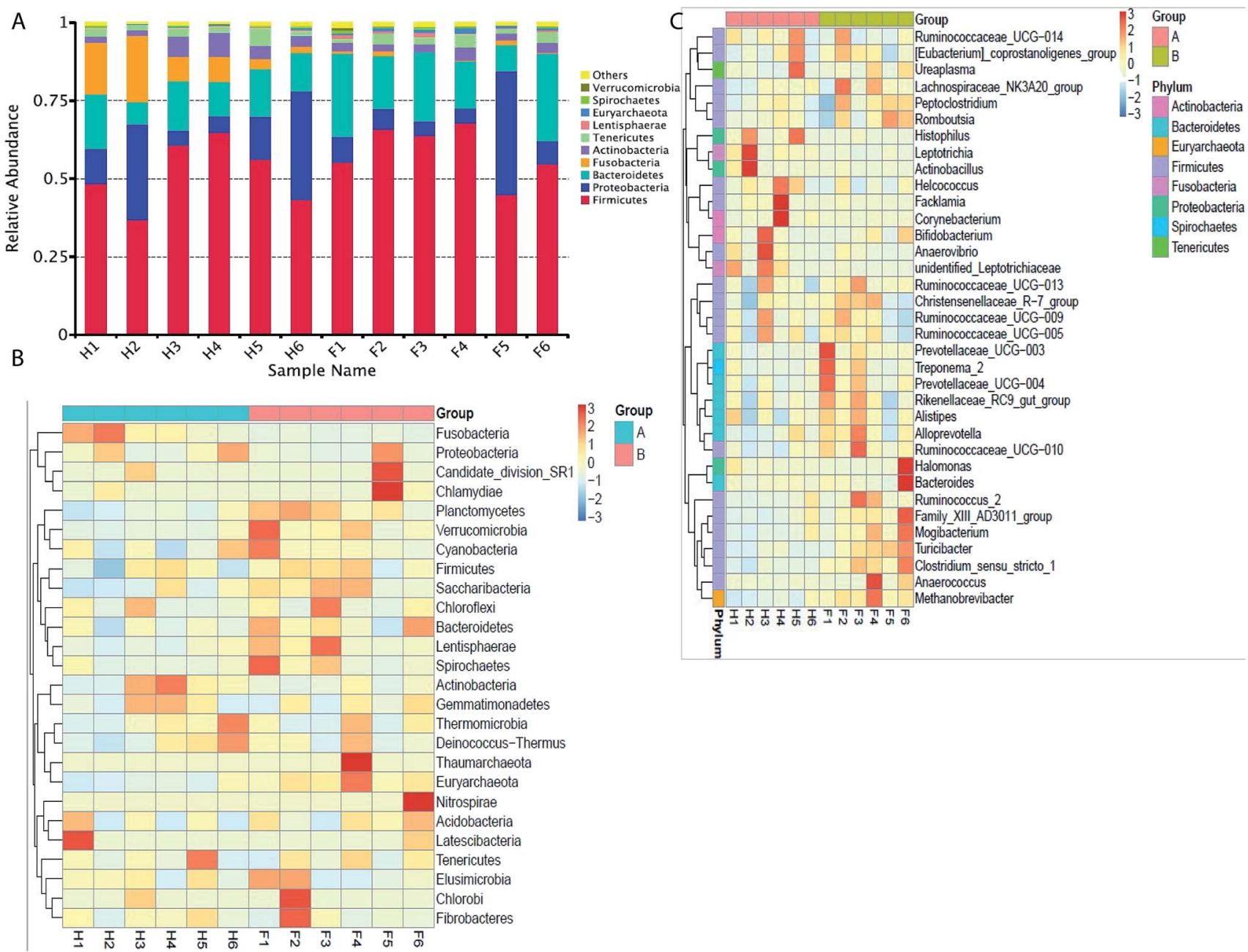

Fig. 3 Taxonomic profiles of the microbial communities of the Holstein and Fleckvieh cattle derived from 16S rRNA gene sequencing of V4 region data. (A) Microbial composition at the phylum level. The samples names are as previously explained. Samples are presented along with the horizontal axis and relative abundance at the vertical axis. (B) Heatmap showing the 26 phyla with significant differences in relative abundances among the two breeds. Heatmap is color-coded based on the scale of -3 to 3. (C) Heatmap showing the 35 genera with significant differences of relative abundances among the two breeds. Heatmap is color-coded based on the scale of -3 to 3 .
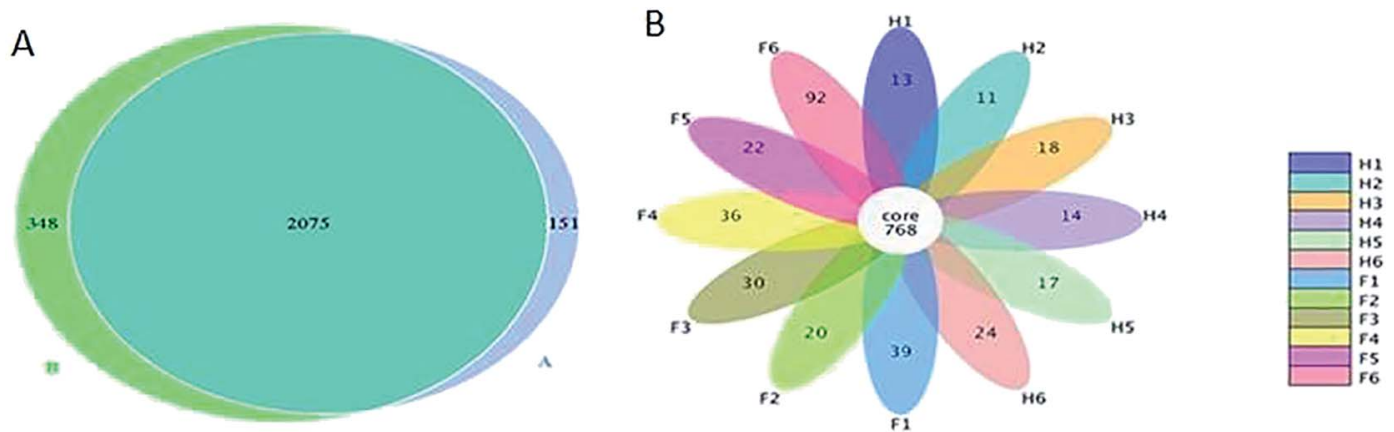

Fig. 4 Comparison of the OTUs in Holstein and Fleckvieh cattle. The number of observed OTUs sharing $\geq 97 \%$ nucleotide sequence identity. (A) A Venn diagram was generated to describe the common and unique OTUs among the two cattle breeds. (B) A flower diagram was used to identify the common and unique OTUs within each individual from the two breeds.

Fleckvieh. Higher abundance of Fusobacteria has been reported to be associated with uterine diseases and related infections. ${ }^{30}$ It was further reported that low Fusobacteria abundance in vaginal microbiota is associated with healthy cows. ${ }^{31}$ From the current observation, the Holstein cattle can be more susceptible to uterine infections than the Fleckvieh cattle. 
The microbial differences were further evaluated at the genus level to gain more insight into vaginal microbial differences between the two cattle breeds. It was found that most of the significantly $(p<0.05)$ different genus species are more abundant and diverse in the Fleckvieh than in the Holstein. A greater difference was also observed at the family level (results not shown) as well as the class level (results not shown) at a similar magnitude, in which Fleckvieh was more in abundance.

The other difference between the two breeds was observed at the OTUs, in which the Fleckvieh cattle had greater unique OTUs than the Holstein cattle. This phenomenon was also observed in individual sequences. The Fleckvieh cattle had more OTUs per individual, while the Holstein had less OTUs per individual sample. The current results indicate that the Holstein cattle have less microbial abundance and OTUs as compared to the Fleckvieh cattle.

\section{Conclusions}

In our study, we have demonstrated, for the first time, the influence of breed type on the vaginal microbial genetic abundance and diversity. The current results are of critical importance for better understanding of vaginal microbial genetic diversity and abundance in different breeds. Such information can be used as a scientific tool to help in reducing and better understanding the physiological challenges in livestock practice to advance reproduction health methods.

\section{Conflicts of interest}

There are no conflicts to declare.

\section{Acknowledgements}

This work was supported by National Natural Science Foundation of China (31672420), Jilin Province key scientific and technical project (20140203016NY; 20140203013NY) and general project of the Department of Science and Technology of Jilin province (20150204074NY; 20150519018JH; 20160209001NY).

\section{Notes and references}

1 A. d. B. des Roches, I. Veissier, X. Boivin, E. Gilot-Fromont and L. Mounier, J. Dairy Sci., 2016, 99, 5573-5585.

2 J. H. H. M. van de Wijgert, H. Borgdorff, R. Verhelst, T. Crucitti, S. Francis, H. Verstraelen and V. Jespers, PLoS One, 2014, 9, e105998.

3 B. Chaban, M. G. Links, T. P. Jayaprakash, E. C. Wagner, D. K. Bourque, Z. Lohn, A. Y. K. Albert, J. van Schalkwyk, G. Reid, S. M. Hemmingsen, J. E. Hill and D. M. Money, Microbiome, 2014, 2, 23.

4 J. Wang, C. Sun, C. Liu, Y. Yang and W. Lu, Anaerobe, 2016, $38,1-6$.
5 C. Gonzalez Moreno, C. Fontana, P. S. Cocconcelli, M. L. Callegari and M. C. Otero, J. Appl. Microbiol., 2016, 121, 1232-1241.

6 K. Aagaard, K. Riehle, J. Ma, N. Segata, T.-A. Mistretta, C. Coarfa, S. Raza, S. Rosenbaum, I. Van den Veyver, A. Milosavljevic, D. Gevers, C. Huttenhower, J. Petrosino and J. Versalovic, PLoS One, 2012, 7, e36466.

7 J. D. Swartz, M. Lachman, K. Westveer, T. O'Neill, T. Geary, R. W. Kott, J. G. Berardinelli, P. G. Hatfield, J. M. Thomson, A. Roberts and C. J. Yeoman, Front. Vet. Sci., 2014, 1, 19.

8 R. F. Lamont, J. D. Sobel, R. A. Akins, S. S. Hassan, T. Chaiworapongsa, J. P. Kusanovic and R. Romero, BJOG, 2011, 118, 533-549.

9 D. N. Fredricks, Anaerobe, 2011, 17, 191-195.

10 J. Handelsman, Microbiol. Mol. Biol. Rev., 2004, 68, 669-685. 11 H. Teeling and F. O. Glöckner, Briefings Bioinf., 2012, 13, 728-742.

12 S. Jiao, H. Cao, Y. Dai, J. Wu, J. Lv, R. Du and B. Han, J. Sci. Food Agric., 2017, DOI: 10.1002/jsfa.8380.

13 S. Mao, M. Zhang, J. Liu and W. Zhu, Sci. Rep., 2015, 5, 16116. 14 R. Roehe, R. J. Dewhurst, C.-A. Duthie, J. A. Rooke, N. McKain, D. W. Ross, et al., PLoS Genet., 2016, 12(2), e1005846.

15 W. Zhao, Y. Wang, S. Liu, J. Huang, Z. Zhai, C. He, et al., PLoS One, 2015, 10(2), e0117441.

16 R. Lamendella, J. W. Santo Domingo, S. Ghosh, J. Martinson and D. B. Oerther, BMC Microbiol., 2011, 11, 103.

17 A. S. Zevin, I. Y. Xie, K. Birse, K. Arnold, L. Romas, G. Westmacott, R. M. Novak, S. McCorrister, L. R. McKinnon, C. R. Cohen, R. Mackelprang, J. Lingappa, D. A. Lauffenburger, N. R. Klatt and A. D. Burgener, PLoS Pathog., 2016, 12, e1005889.

18 W.-L. Wang, S.-Y. Xu, Z.-G. Ren, L. Tao, J.-W. Jiang and S.-S. Zheng, World J. Gastroenterol., 2015, 21, 803-814.

19 D. A. MacIntyre, M. Chandiramani, Y. S. Lee, L. Kindinger, A. Smith, N. Angelopoulos, B. Lehne, S. Arulkumaran, R. Brown, T. G. Teoh, E. Holmes, J. K. Nicoholson, J. R. Marchesi and P. R. Bennett, Sci. Rep., 2015, 5, 8988.

20 M. Laguardia-Nascimento, K. M. G. R. Branco, M. R. Gasparini, S. Giannattasio-Ferraz, L. R. Leite, F. M. G. Araujo, et al., PLoS One, 2015, 10(11), e0143294.

21 T. Magoc and S. L. Salzberg, Bioinformatics, 2011, 27, 29572963.

22 J. G. Caporaso, J. Kuczynski, J. Stombaugh, K. Bittinger, F. D. Bushman, E. K. Costello, N. Fierer, A. G. Pena, J. K. Goodrich, J. I. Gordon, G. A. Huttley, S. T. Kelley, D. Knights, J. E. Koenig, R. E. Ley, C. A. Lozupone, D. McDonald, B. D. Muegge, M. Pirrung, J. Reeder, J. R. Sevinsky, P. J. Turnbaugh, W. A. Walters, J. Widmann, T. Yatsunenko, J. Zaneveld and R. Knight, Nat. Methods, 2010, 7, 335-336.

23 Q. Wang, G. M. Garrity, J. M. Tiedje and J. R. Cole, Appl. Environ. Microbiol., 2007, 73, 5261-5267.

24 R. C. Team, $R$ : A language and environment for statistical computing, $\mathrm{R}$ Foundation for Statistical Computing, Vienna, Austria, 2014, http://www.R-project.org/. 
25 R. J. Wallace, J. A. Rooke, N. McKain, C.-A. Duthie, J. J. Hyslop, D. W. Ross, A. Waterhouse, M. Watson and R. Roehe, BMC Genomics, 2015, 16, 839.

26 A. Kumar and D. Kumar, Anaerobe, 2015, 33, 117-123.

27 V. S. Machado, G. Oikonomou, M. L. S. Bicalho, W. A. Knauer, R. Gilbert and R. C. Bicalho, Vet. Microbiol., 2012, 159, 460-469.

28 E. Jami and I. Mizrahi, PLoS One, 2012, 7, e33306.
29 S. F. Lima, A. G. V. Teixeira, F. S. Lima, E. K. Ganda, C. H. Higgins, G. Oikonomou and R. C. Bicalho, J. Dairy Sci., 2017, 100, 3031-3042.

30 M. L. S. Bicalho, T. Santin, M. X. Rodrigues, C. E. Marques, S. F. Lima and R. C. Bicalho, J. Dairy Sci., 2017, 100, 30433058.

31 M. L. S. Bicalho, S. Lima, C. H. Higgins, V. S. Machado, F. S. Lima and R. C. Bicalho, J. Dairy Sci., 2017, 100, 38633874 . 